\title{
Title: Encoding sensing functions into material interface for a rationally engineered integrated electrochemical liquid biopsy
}

Authors: Yuan Zhang ${ }^{1, *,}$, Hao Zhu ${ }^{1, \dagger}$, Zi Ying ${ }^{1}$, Xinghua Gao', Wei Chen², Yueping Zhan ${ }^{3}$, Lingyan Feng ${ }^{1}$, Chung-Chiun Liü \& Yifan Dai ${ }^{5, *}$

Affiliations:

${ }^{1}$ Materials Genome Institute, Shanghai University, Shanghai 200444, China

${ }^{2}$ Department of Emergency, Tongji Hospital, Tongji University School of Medicine, Shanghai 200065, China

${ }^{3}$ Interventional Cancer Institute of Chinese Integrative Medicine, Shanghai University of Traditional Chinese Medicine, Shanghai, 200062, China

${ }^{4}$ Department of Chemical and Biomolecular Engineering, Case Western Reserve University, Cleveland, $\mathrm{OH} 44106$, USA

${ }^{5}$ Department of Biomedical Engineering, Duke University, Durham, NC 27705, USA

tThese authors contributed equally to this work.

${ }^{*}$ Correspondence to Yuan Zhang (email: zhangyuan@shu.edu.cn) and Yifan Dai (email: yifan.dai1@duke.edu).

\begin{abstract}
Limited healthcare capacity highlights the needs of integrated and simple sensing systems for personalized health monitoring. However, only a limited set of sensors can be employed for point-of-care applications, emphasizing the lack of a generalizable engineering strategy for sensor construction. Here, we report a de novo rational engineering strategy for the construction of an integrated electrochemical liquid biopsy (ELB) platform capable of direct profiling cancer exosomes from blood. Using a bottom-up approach for sensor design, a series of critical sensing functions is considered and encoded into the material interface by programming the electrode material with different chemical and structure features. We present that the rationally engineered electrochemical liquid biopsy platform is able to achieve one-step sensor fabrication, target isolation, non-fouling and high-sensitivity sensing, direct signal transduction and multiplexed detection. Integrating the multiplexed sensing with principal component analysis, we demonstrate the capability of the programmed sensing system on differentiating cancerous groups from healthy controls by analyzing clinical samples from lung cancer patients.
\end{abstract}

\section{Key words}

Rationally designed sensor, de novo programming sensing materials, metal-organic framework, electrochemical liquid biopsy, point-of-care system, fully integrated sensor, cancer diagnosis 


\section{Introduction}

The ongoing severe acute respiratory syndrome coronavirus 2 (SARS-CoV-2) pandemic poses an imminent healthcare challenge to the world. The high infectivity, long incubation time and asymptomatic nature of SARS-CoV-2 result widespread transmission across the world with over 310 million cases ${ }^{1}$. As a result, current healthcare capacities are overwhelmed across the world, significantly impacting the timely diagnostics and treatments of patients with other types of severe diseases ${ }^{2}$, especially metastatic cancers ${ }^{3}$. Such unexpected scenarios highlight the long-term needs of simple, reliable, and accurate diagnostic systems for metastatic cancer management.

Liquid biopsy, which detects tumor biomarkers directly from blood sample, is the current gold-standard for non-invasive metastatic cancer managements ${ }^{4}$. However, the analytical methods applied in liquid biopsy include enzyme-linked immunosorbent assays (ELISA) for protein analysis and next-generation sequencing for nucleic acid analysis ${ }^{5}$, which require laboratory settings, technical expertise and long turnaround time to achieve reliable diagnostic, limiting its applicability in resource-limited areas, especially in current unusual time.

To tackle the challenges for the developments of a simple, quantitative, and accurate assay toward detecting metastatic cancers, we herein present a de novo engineering strategy for the development of electrochemical liquid biopsy (ELB) platform, enabling direct sorting and multiplexed analysis of different biomarkers on tumor-derived extracellular vesicles. By encoding critical sensing functions into the material interfaces, the developed ELB is capable of direct quantitative detection of cancers from a single drop of blood within $30 \mathrm{~min}$. ELB was employed for the analysis of blood from patients with lung cancer, showing excellent correlation with clinically established cancerous group. Owing to the modularity of our demonstrated engineering approach, the ELB can be programmed to a generalizable platform technology for the detection of other different biomarkers.

\section{Results}

\section{Rational engineered hybrid nanostructured electrode as a sensing interface}

For the development of multiplexed, point-of-care ELB, we rationally evaluated the technical criteria that are imperative to enable a fully integrated ELB system (Fig. 1). Specifically, for the purpose of direct analysis of cancer related extracellular vesicles, the following sensing features should be encoded into the sensing interface: 1) efficient electron transfer ensuring high signal-to-noise ratio to achieve an ideal detection sensitivity (i.e. detection limit) and sensing resolution (i.e. signal differentiation), 2) specific target sorting from complex biological samples, 3) non-fouling surface ensuring detection sensitivity in complex biological matrix, 4) direct target sensing with limited operational procedures, 5) multiplexed analysis guaranteeing the high-accuracy of the platform. To achieve these desired functions, we de novo designed and constructed a miniaturized and mass-producible nano-structured sensor system through programming the material properties of the hybrid nanocomposites. 


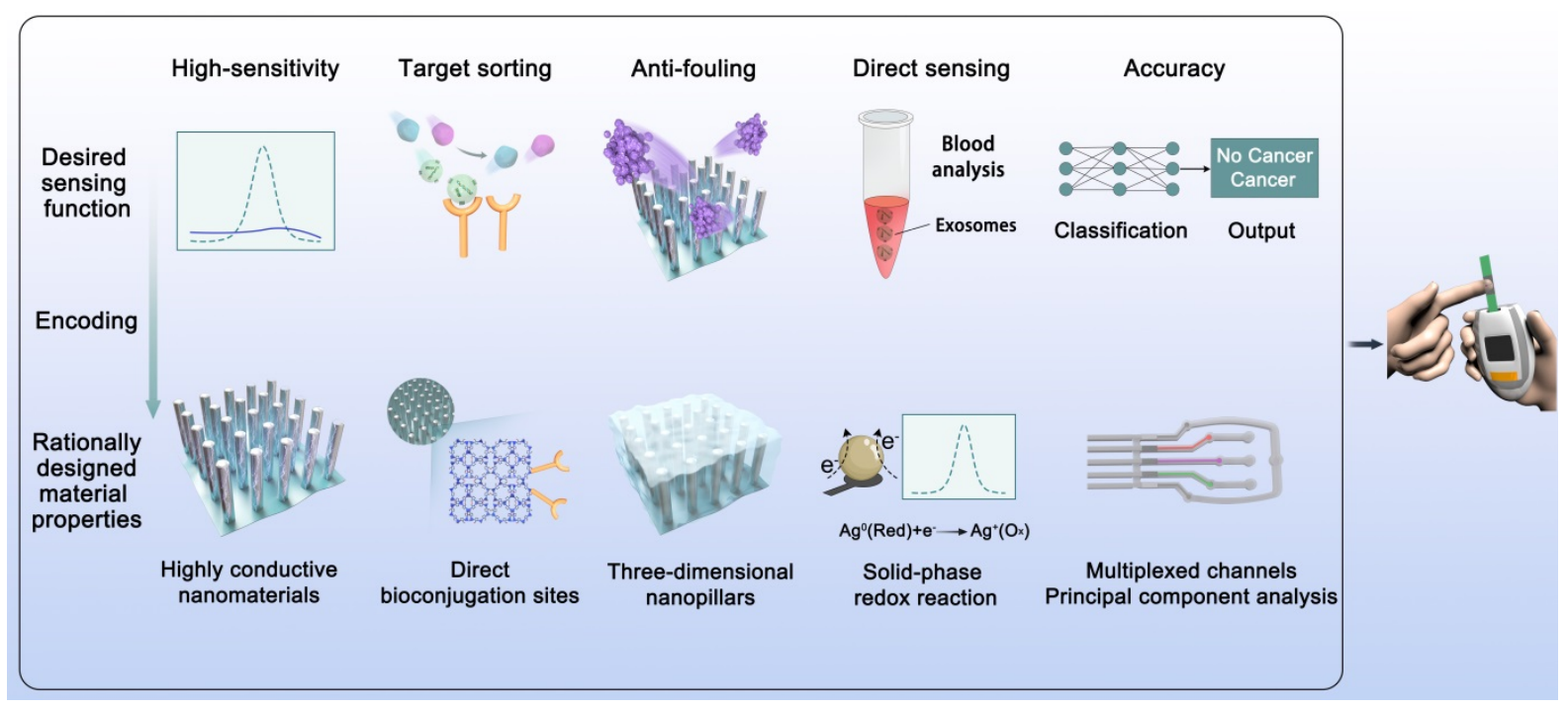

Figure. 1 Schematic illustration of the rational engineering strategy toward the development of electrochemical liquid biopsy. Desired sensing functions serve as the criteria for the design of sensing interface. Through bottom-up programming and constructing the material properties of the sensing interface, functional sensing features are encoded. This rational engineering process delivers a fully integrated electrochemical liquid biopsy platform technology.

To construct a sensing interface enabling a high signal-to-noise ratio, we employed a nanostructured pillar array providing efficient mass transport and electron transfer channels for signal amplification ${ }^{6}$. Specifically, ordered $\mathrm{ZnO}$ nanopillar was grown onto ultrathin $1 \mathrm{~T} \mathrm{MoS} 2$ nanosheets forming a hybrid nanostructure. In order to achieve exosomes separation from blood, we applied a ZIF-90 thin film containing aldehyde group (-CHO) for bioconjugation to immobilize recognition antibodies of exosome proteins through direct interacting with $-\mathrm{NH}_{2}$ groups on the antibodies. In the ZIF-90 thin film, abundant $-\mathrm{CHO}$ groups are periodically arranged in the rigid metal-organic framework ${ }^{7}$, avoiding the potential degradation of antibody by using conventional flexible glutaraldehyde molecule ${ }^{8}$. Target bioconjugation can induce the desired conformation of antibody ${ }^{9,10}$, and would be in favor of the maximized capture efficiency of exosomes through affinity binding. Furthermore, such three-dimensional (3D) porous matrix, by simply interlacing with bovine serum albumin (BSA), can achieve anti-fouling property ${ }^{11}$. To achieve direct quantification, we took advantages of the customized electrodes, in which solid-phase electroactive species (silver/carbon composite) were paved underneath the nano-structured electrode for redox reaction ${ }^{12}$. Therefore, with the presence of the highly negatively charged exosomes ${ }^{13}$ on the surface, the electron transfer process would be significantly hindered, realizing quantitative difference in electrochemical current. Comparing with conventional electrochemical quantification methods through investigating the change of surface impedance ${ }^{14}$, by encoding the redox reaction into the materials, we eliminated the use of typical electrochemical redox molecules ${ }^{14}$ (e.g. ferricyanide or ruthenium nitride) which can potentially cause irritation of eyes and skins, providing a more user-friendly and safe sensing system. Finally, to fulfill the need of high-accuracy exosomes analysis, we designed a three-channel microfluidic sensor chip, enabling simple preparation process of ELB and multiplexed simultaneous sensing. To test the capability of this platform, we selected CD9, CD63 and CD81 $1^{15,16}$ as the detecting targets, which residue on the surface of the cancer related extracellular vesicles and have been clinically validated for their efficacy. To prevent false-positive/negative results, 
bioRxiv preprint doi: https://doi.org/10.1101/2022.01.17.476350; this version posted January 20, 2022. The copyright holder for this preprint (which was not certified by peer review) is the author/funder, who has granted bioRxiv a license to display the preprint in perpetuity. It is made available under aCC-BY-NC-ND 4.0 International license.

we employed principal component analysis to process the electrochemical data, maximizing sample variations.

\section{Design of microsensor chip enables direct signal transduction}

The microsensor chip is composed of screen-printed electrodes sealed with polydimethylsiloxane (PDMS) layer. Five electrodes, including counter electrode (CE), reference electrode (RE) and three different working electrodes (WE), are printed on a polyethylene terephthalate (PET) substrate (See methods for details, Supplementary Fig. 1). To enable direct electrochemical sensing without external introduction of redox active chemicals, we first evaluated whether electrochemical redox reaction can be encoded into the solid-phase materials. We evaluated whether the embedded silver/carbon electrical conduction band underneath the WE is capable of generating electrochemical signal, owing to the known $\mathrm{Ag}^{0} / \mathrm{Ag}^{+}$redox reaction ${ }^{17,18}$. By applying cyclic voltammetry (CV) in phosphate buffer, a pair of redox peaks corresponding to consecutive one-electron-transfer reactions of $\mathrm{Ag}^{0}(\operatorname{Red}) / \mathrm{Ag}^{+}(\mathrm{Ox})$ $\left(\mathrm{E}_{\mathrm{Ox}}=0.08 \mathrm{~V}\right.$ vs. $\left.\mathrm{Ag} / \mathrm{AgCl}\right)$ and $\mathrm{Ag}^{+}(\mathrm{Ox}) / \mathrm{Ag}^{0}(\mathrm{Red})\left(\mathrm{E}_{\mathrm{Red}}=-0.13 \mathrm{~V}\right.$ vs. $\left.\mathrm{Ag} / \mathrm{AgCl}\right)$ was observed (Supplementary Fig. 2), confirming our capability to encode redox reaction into solid-phase materials.

\section{Characterizations of rationally engineered nanostructured sensing material}

Based on the considerations set for an ideal sensing interface toward electrochemical analysis, we designed and engineered the metal organic framework $\mathrm{MoS}_{2} / \mathrm{ZnO} / \mathrm{ZIF}-90$ based nanostructure as the materials of working electrode through hierarchical growth (see Methods) to achieve robust electron transfer and low-matrix effect ${ }^{11}$. Material and surface characterizations were performed to evaluate the chemical identity and structure of the designed material.

First, SEM images of $\mathrm{MoS}_{2} / \mathrm{ZnO}$ sample show that the ordered nanopillars array is uniformly grown onto ultrathin $\mathrm{MoS}_{2}$ nanosheets (Fig. 2a). The high-magnification of SEM image reveal that the diameter of vertical $\mathrm{ZnO}$ nanopillars is $20-40 \mathrm{~nm}$ and a glazed surface is observed over nanopillars (Fig. 2b). After exposure to imidazole-2-carboxaldehyde (2-ICA) vapor, the surface of $\mathrm{ZnO}$ nanopillars became roughened with bumpy and hollow structures (Fig. 2c). Elemental mapping analysis in nanopillars region demonstrates the homogeneous distribution of $\mathrm{Zn}, \mathrm{O}, \mathrm{C}$ and $\mathrm{N}$ species (Fig. 2d). It should be noted that the contents of $\mathrm{C}$ and $\mathrm{N}$ are slightly lower than that of another two species, indicating that only the surface portion of $\mathrm{ZnO}$ nanopillars was turned into ZIF-90. The low-magnification TEM image shows that the length of nanopillars is about $1.5 \mu \mathrm{m}$ (Fig. 2e), and further HRTEM characterization demonstrates that the nanopillars are core-shell structure with 8-12 $\mathrm{nm}$ of ZIF-90 layer formed on the surface (Fig. 2f). During the growth of ZIF-90 thin layer, we found the reaction time is critical for the formation and structure. With optimized reaction time, the integrated $\mathrm{MoS}_{2} / \mathrm{ZnO} / \mathrm{ZIF}-90$ nanoarchitecture is successfully prepared (Supplementary Fig. 3).

Furthermore, to evaluate the morphological feature of the complex nanostructure, we applied electron microscope tomography. The micrographs of $\mathrm{MoS}_{2} / \mathrm{ZnO} / \mathrm{ZF}-90$ sample were recorded from various orientations by tilting the specimen, which were then computationally merged into a $3 \mathrm{D}$ structure (Supplementary Fig. 4). According to these images, ultrathin $\mathrm{MoS}_{2}$ material displayed as blue color is located at the bottom of the $3 \mathrm{D}$ nanoarchitecture, and the outermost green is originated from ZIF-90 thin film. Additionally, the red $\mathrm{ZnO}$ nanopillars are appeared to be orange due to its overlapping with the outer green thin film. These electron microscopy 2D and 3D images provide the detailed structure 

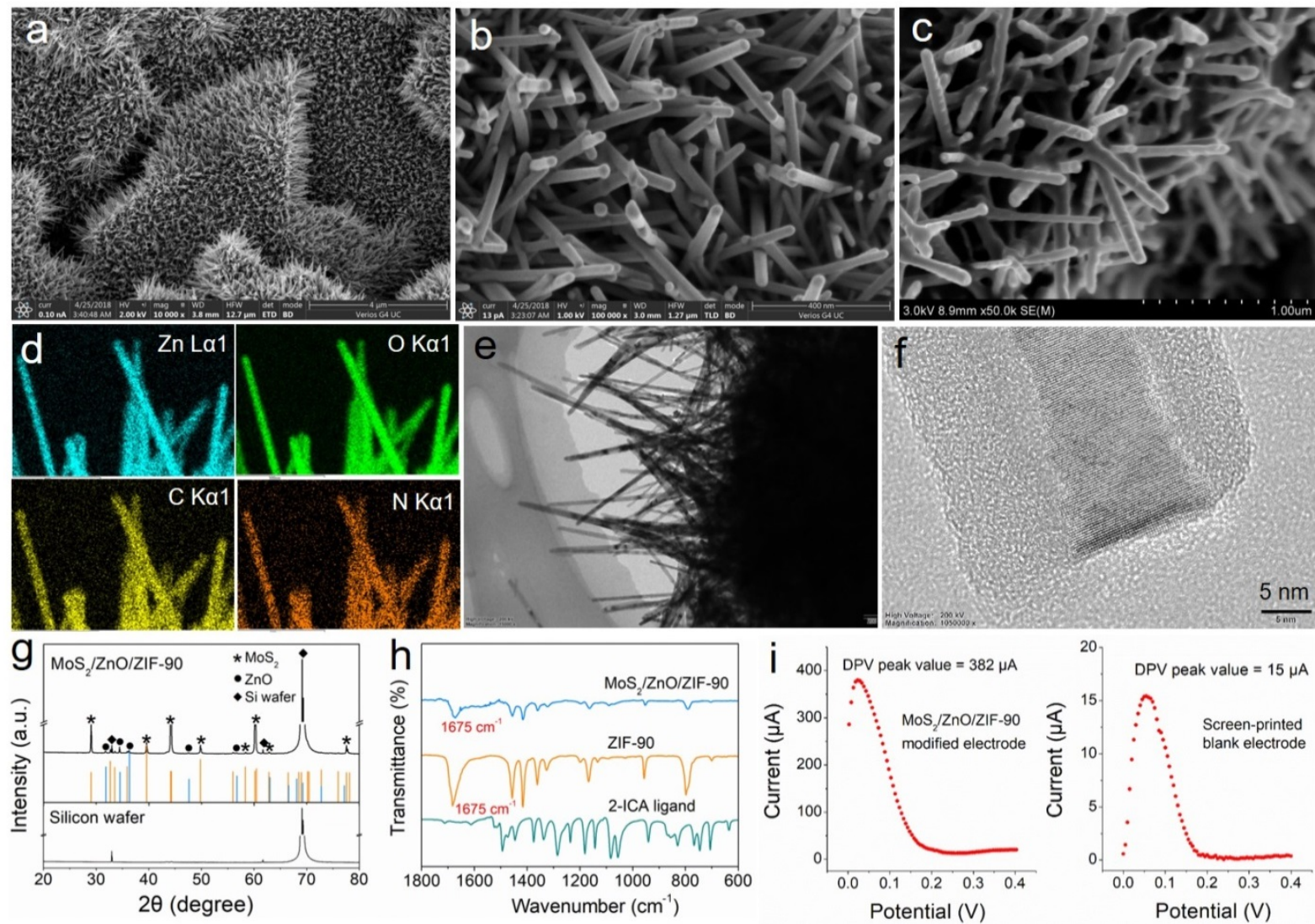

Figure. 2 Characterizations of $\mathrm{MoS}_{2} / \mathrm{ZnO}$ and $\mathrm{MoS}_{2} / \mathrm{ZnO} / \mathrm{ZIF}-90$ nanostructures based working electrode and the configuration of fabricated microsensor. $\mathbf{a}, \mathbf{b}$, Low-magnification and high-magnification SEM images of $\mathrm{MoS}_{2} / \mathrm{ZnO}$. c, High-magnification SEM image of $\mathrm{MoS}_{2} / \mathrm{ZnO} / \mathrm{ZIF}-90$. d, Elemental mapping analysis of $\mathrm{MoS}_{2} / \mathrm{ZnO} / Z I F-90$. e, TEM image of $\mathrm{MoS}_{2} / Z n O / Z I F-90$. f, HR-TEM images of $\mathrm{MoS}_{2} / Z n O / Z I F-90 . \mathbf{g}$, XRD pattern of $\mathrm{MoS}_{2} / \mathrm{ZnO} / \mathrm{ZIF}-90$. h, FT-IR spectra of $\mathrm{MoS}_{2} / \mathrm{ZnO} / \mathrm{ZIF}-90, \mathrm{ZIF}-90$ and imidazole-2-carboxaldehyde. i, Programmed materials mediated amplification of signal amplification as demonstrated through measurements by differential pulse voltammetry on $\mathrm{MoS}_{2} / \mathrm{ZnO} / \mathrm{ZIF}-90$ modified or unmodified screen-printed electrode in PBS solution $(\mathrm{pH}=7.2)$.

Moreover, X-ray diffraction (XRD) characterization shows the pattern consistency between the fabricated $\mathrm{MoS}_{2}$ with standard $2 \mathrm{H}-\mathrm{MoS}_{2}$ structure (JCPDS No. 03-065-0160), and the peaks labeled with black dots are corresponding to the strongest peaks of ZnO (JCPDS No. 01-075-0576) (Fig. 2g). No peak from ZIF-90 was observed because only a thin layer of $\mathrm{ZnO}$ is consumed and transformed into ZIF-90. Fourier-transform infrared spectroscopy (FT-IR) was then performed (Fig. 2h) and all the peaks in the spectrum of $\mathrm{MoS}_{2} / \mathrm{ZnO} / \mathrm{ZIF}-90$ sample are in excellent agreement with that of pure ZIF-90 and no characteristic peak derived from 2-ICA ligand is present, indicating the successful transformation of 2-ICA to ZIF-90 instead of simple surface absorption. The bands in the spectral region of $600-1500 \mathrm{~cm}^{-1}$ are associated with the stretching and bending modes of the imidazole ring $^{19,20}$. Most importantly, the characteristic peak at about $1675 \mathrm{~cm}^{-1}$ reveals the presence of $-\mathrm{CHO}$ in materials ${ }^{21,22}$, confirming our capability on direct immobilization of recognition elements. These 
bioRxiv preprint doi: https://doi.org/10.1101/2022.01.17.476350; this version posted January 20, 2022. The copyright holder for this preprint (which was not certified by peer review) is the author/funder, who has granted bioRxiv a license to display the preprint in perpetuity. It is made available under aCC-BY-NC-ND 4.0 International license.

characterizations confirmed the chemical identity and structure of the programmed sensing materials.

To test whether such nanostructured electrode enhances electron transfer, we applied differential pulse voltammetry (DPV) to compare the electrochemical signal before and after the addition of $\mathrm{MoS}_{2} / \mathrm{ZnO} / \mathrm{ZIF}-90$ layer. The peak current of modified electrode leads to a $2446.7 \%$ increase comparing with unmodified electrode (Fig. 2i), confirming capability of signal amplification by the developed nanostructured electrode ${ }^{23,24}$.

\section{Microfluidic channels enable simple sensor fabrication and multiplexed sample analysis}

To achieve multiplexed analysis, we set out to integrate multi-channel microfluidics as the sample inlets for the electrochemical microsensor (Fig. 3a). The microchannels on the chip can be employed for the immobilization of bio-recognition antibodies for sensor fabrication as well as simultaneous multiplexed detection of exosome markers.

It is critical to ensure the sealing ability and orthogonality of microchannels for processing liquid samples. To test this notion, solutions containing different dyes were added into the microchannels and no leakage was observed (Supplementary Fig. 5). We further introduced different dyes separately into three microchannels from their corresponding inlets, demonstrating orthogonality on liquid transportation. Therefore, these channels can be directly applied to immobilize Anti-CD9, Anti-CD63 and Anti-CD81 antibodies onto WE-1, WE-2 and WE-3 respectively, presenting a simple procedure for sensor fabrication (See methods for details and Supplementary Fig. 1).

For the purpose to achieve direct multiplexed analysis of sample, a fourth inlet was designed to accommodate testing sample, allowing flow diversion into all the WEs. To ensure sensing accuracy, we tested the consistency of output signals through different microchannels. The phosphate buffered saline (PBS) solution $(\mathrm{pH}=7.2,100 \mu \mathrm{L})$ was filled into the chip channels, and the obtained CV curves overlap with each other (Supplementary Fig. 6), indicating that the engineering and fabrication techniques are reliable to produce reproducible sensing interface.

\section{Characterizations of the ZIF-90 for immobilization of recognition elements}

For the purpose of target sorting and detection of exosomes, the prepared $\mathrm{MoS}_{2} / \mathrm{ZnO} / \mathrm{ZIF}-90$ material was tested for antibody immobilization and the following immunoreaction. A fluorescein isothiocyanate (FITC) labeled antibody was employed and the confocal fluorescence microscope was used to observe the results of immobilization. Here, FITC Anti-CD63 is served as an illustration. We applied WE-1 and WE-3 pre-deposited with MoS $2 / \mathrm{ZnO} / \mathrm{ZIF}-90$ and WE-2 (located in the middle of the sensor chip) pre-deposited with only $\mathrm{MoS}_{2} / \mathrm{ZnO}$ material for the immobilization of FITC Anti-CD63. Comparing the dark field, bright field and merged fluorescence images of different WEs (Fig. 3b-d), two clearly defined spots were noticed in the region where the electrodes are covered by $\mathrm{MoS}_{2} / \mathrm{ZnO} / \mathrm{ZIF}-90$ material, while no change in fluorescence intensity was observed along the middle channel. The 3D fluorescence images also show that the uniform green fluorescence is appeared over $\mathrm{MoS}_{2} / \mathrm{ZnO} / \mathrm{ZIF}-90$ material, while there is almost no fluorescence observed by using $\mathrm{MoS}_{2} / \mathrm{ZnO}$ as electrode material (Fig. 3e-f). Moreover, a vertically array structure is dimly visible in the whole observation area, further revealing that FITC Anti-CD63 is immobilized on the surface of nanopillars, potentially enhancing the target capture capability with such $3 \mathrm{D}$ structure ${ }^{25}$. These fluorescence 


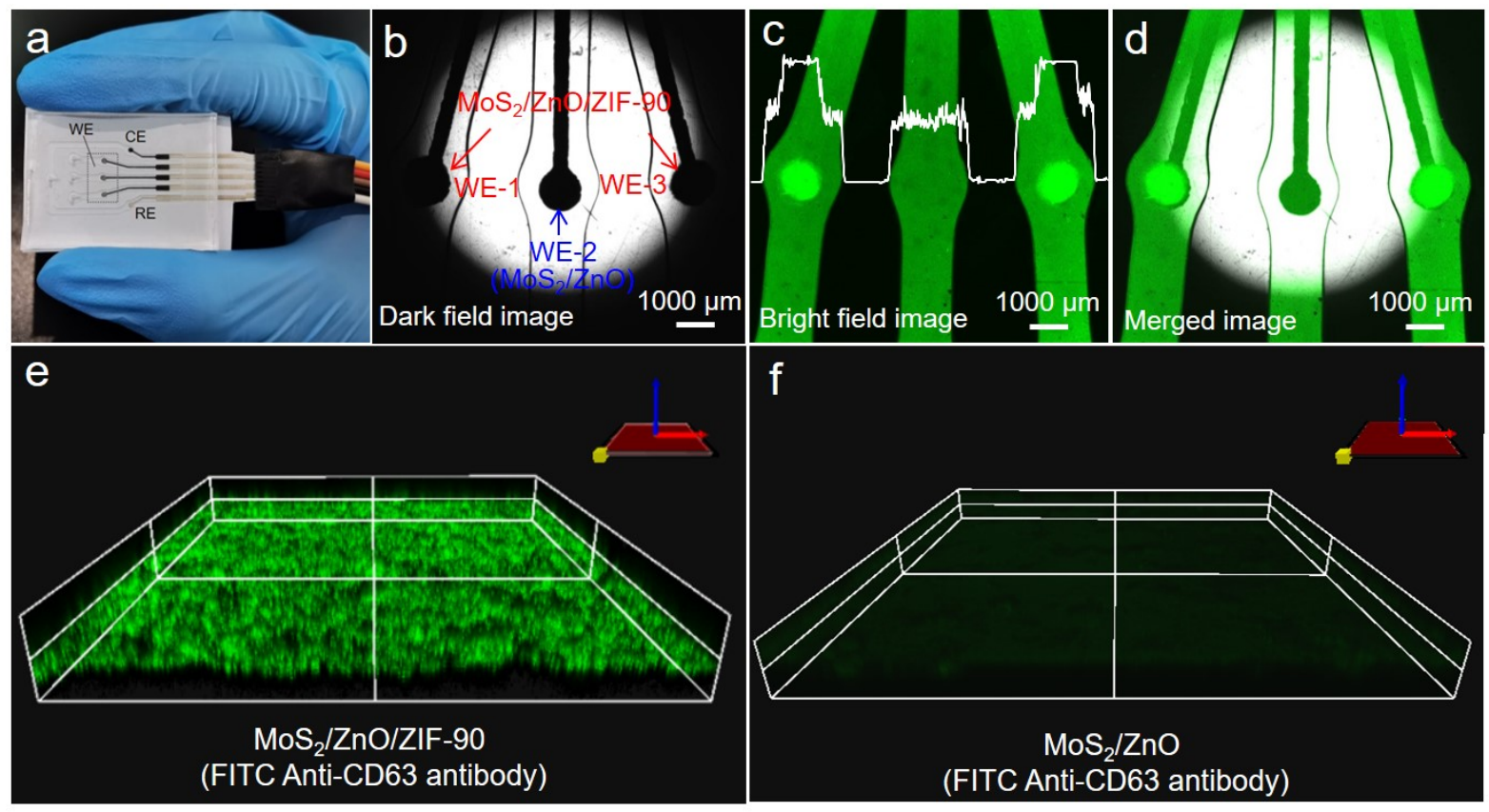

Figure 3. Direct immobilization of recognition elements onto the nanopillar. a, An integrated three-channel sensor chip (WE=working electrode, $\mathrm{CE}=$ counter electrode and $\mathrm{RE}=$ reference electrode). b, c, d, Dark field, bright field and merged fluorescence images of different WEs. Two of WEs (WE-1 and WE-3) are deposited with $\mathrm{MoS}_{2} / \mathrm{ZnO} / \mathrm{ZIF}-90$ nanohybrids, and WE-2 is loaded with $\mathrm{MoS}_{2} / \mathrm{ZnO}$ nanomaterial. All three WEs are incubated with FITC Anti-CD63 antibody. e, f, 3D fluorescence images of $\mathrm{MoS}_{2} / \mathrm{ZnO} / \mathrm{ZIF}-90$ and $\mathrm{MoS}_{2} / \mathrm{ZnO}$ after immobilized with FITC Anti-CD63 antibody.

characterizations confirm that $\mathrm{MoS}_{2} / \mathrm{ZnO} / \mathrm{ZIF}-90$ nanostructure could be used for the effective immobilization of antibody depending on the surface ZIF-90 thin film. To verify the antibody immobilization is solely attributed to ZIF-90 instead of a cooperative effect of the nanostructures, we employed ZIF-90 nanoparticles for antibody immobilization (Supplementary Fig. 7). Similarly, strong fluorescence intensity is also detected over ZIF-90 nanoparticles. Although pure ZIF-90 nanoparticles possess the ability to effectively immobilize recognition antibody, only a minute current response was measured (Supplementary Fig. 8). This control experiment illustrates the importance of the synergistic effect of the sensing materials on sensing functions. 

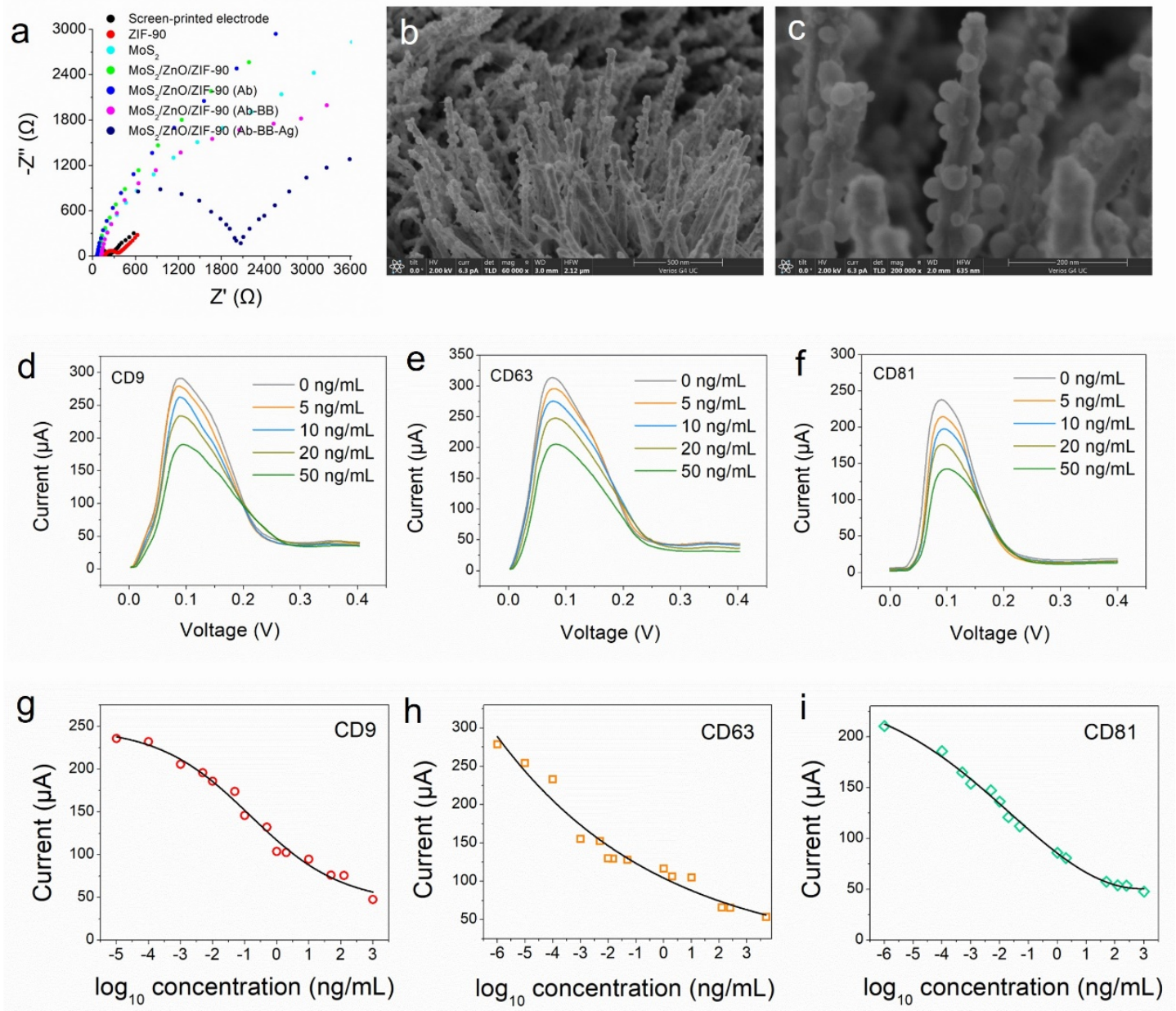

Figure 4. Characterizations of the programmed materials on target sorting and quantitative sensing. a, EIS measurement (Nyquist plots) of different materials (ZIF-90, $\mathrm{MoS}_{2}$ and $\mathrm{MoS}_{2} / \mathrm{ZnO} / \mathrm{ZIF}-90$ ) as well as the developed microsensor during the preparation process. b, SEM image showing the uniformly captured exosomes on the surface nanopillars. c, High-magnification SEM image revealing the size and morphology of exosomes. d, e, f, DPV measurements of the developed microsensor in the presence of CD9 (WE-1), CD63 (WE-2) and CD81 (WE-3) at various concentrations. g, h, i, Dose-dependent responses of exosomes from culture supernatant based on the detection of CD9, CD63 and CD81.

\section{EIS confirms the principles of the nanostructured electrodes toward high-sensitivity analysis and immunoreactions}

The electrochemical impedance spectroscopies (EIS) of different materials as well as the developed microsensor for immunoassay were measured (Fig. 4a) and displayed with an impedance order of silver/carbon screen-printed electrode, ZIF-90, $\mathrm{MoS}_{2}, \mathrm{MoS}_{2} / \mathrm{ZnO} / \mathrm{ZIF}-90, \mathrm{MoS}_{2} / \mathrm{ZnO} / \mathrm{ZIF}-90-A n t i \mathrm{CD} 63$ ( $\mathrm{MoS}_{2} / \mathrm{ZnO} / \mathrm{ZIF}-90-\mathrm{Ab}$ ), $\mathrm{MoS}_{2} / \mathrm{ZnO} / \mathrm{ZIF}-90-A n t i$ CD63- blocking buffer ( $\left.\mathrm{MoS}_{2} / \mathrm{ZnO} / \mathrm{ZIF}-90-\mathrm{Ab}-\mathrm{BB}\right)$, and $\mathrm{MoS}_{2} / \mathrm{ZnO} / \mathrm{ZIF}-90-A n t i$ CD63- blocking buffer-CD63 protein (MoS $/$ ZnO/ZIF-90-Ab-BB-Ag). Generally, EIS is an important electrochemical technique based on the interfacial reaction at the electrode surface, which could be used to evaluate the electron transfer resistance $\left(R_{\mathrm{et}}\right)$ on electrode surface ${ }^{26,27}$. The EIS result is often represented by the Nyquist plot, including a semicircle at high-frequency and linear part at low-frequency region. Since the diameter of semicircle in Nyquist plot is related to $\mathrm{Ret}^{28,29}$, the 
EIS curve of $\mathrm{MoS}_{2}$ shows the disappearance of semicircle at high-frequency region compared with silver/carbon screen-printed electrode, demonstrating that the electron transfer rate is enhanced with the modification of $\mathrm{MoS}_{2}$. However, a clear semicircle was obtained over ZIF-90 modified electrode, which reveals the large $R_{\text {et }}$ of ZIF-90 particles. After modification of $\mathrm{MoS}_{2} / \mathrm{ZnO} / \mathrm{ZIF}-90$ material, only the linear part is appeared and the linear slop is higher than that of pure $\mathrm{MoS}_{2}$, which indicates that the hybrid nanostructure is more favorable for electron transfer than $2 \mathrm{D} \mathrm{MoS} 2$ nanosheets. The Nyquist graphs and fitted values show that the most significant change occurs in $R_{\mathrm{et}}$, and the $R_{\mathrm{et}}$ values of these systems are $61.78 \Omega$ (screen-printed electrode), $156.70 \Omega$ (ZIF-90 modified electrode), $34.43 \Omega$ ( $\mathrm{MoS}_{2}$ modified electrode) and $15.73 \Omega\left(\mathrm{MoS}_{2} / \mathrm{ZnO} / \mathrm{ZIF}-90\right)$, respectively. These results also suggest that the developed nanostructure with an excellent electron transfer capability is beneficial to signal amplification for our sensor system.

Followed by incubation of antibody with $\mathrm{MoS}_{2} / \mathrm{ZnO} / \mathrm{ZIF}-90$, the Nyquist plot appears limited variation, due to the low incubation concentration and neutral charge property of the antibodies used. However, once we blocked the electrode surface with BSA, a depressed semicircle of Nyquist curve was observed. After the final immunoreaction, an obvious semicircle at the high frequency range emerged (Fig. 4a), implying the capability of the developed microsensor on electrochemical immunodetection of exosomes biomarker. The distinct semicircle from the EIS curve of $\mathrm{MoS}_{2} / \mathrm{ZnO} / \mathrm{ZIF}-90-\mathrm{Ab}-\mathrm{BB}-\mathrm{Ag}$ is ascribed to the formation of antibody-antigen complex which impedes the electron transfer on the surface of the electrode. From the above observations, we demonstrated that $\mathrm{MoS}_{2} / \mathrm{ZnO} / \mathrm{ZIF}-90$ nanohybrids not only possess the capability of enhancing electron transfer, but also feasible for immobilization of antibody as well as the following antigen-antibody interaction.

\section{Direct capture of extracellular vesicles from complex matrix}

We then verified the exosomes sorting ability of the microsensor chip through antibody-antigen affinity reaction. By introducing complex biological matrix containing extracellular vesicles onto the sensor, in the presence of a recognition antibody (Anti-CD63), all the nanopillars are uniformly surrounded with exosome particles (Fig. 4b), demonstrating effectiveness of our platform for exosomes capture. The high-magnification SEM image reveals that the diameter of captured exosomes is about $15 \mathrm{~nm}$ to 50 $\mathrm{nm}$ (Fig. 4c). Furthermore, the exosomes are enriched on the surface of the nanopillars, presenting a direct confirmation of that the significantly decreased electron transfer kinetics (Fig. 4a) were due to the presence of negatively charged vesicles.

\section{Verification of electrochemical sensing principle}

For the multiplexed measurement of exosomes markers, we separately introduced different antibodies (Anti-CD9, Anti-CD63 and Anti-CD81) into the corresponding microchannels to construct the immunoelectrodes. Recombinant CD9, CD63 and CD81 proteins with different concentrations (prepared in PBS buffer) were added into three microchannels and incubated at $37{ }^{\circ} \mathrm{C}$ for $30 \mathrm{~min}$. The DPV current responses of different exosomes markers decrease along with the concentrations (Fig.

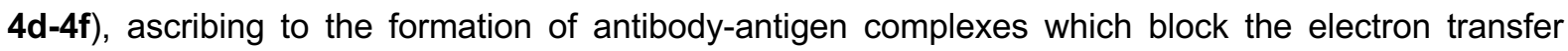
between the WE and CE. These results demonstrate that the engineered microsensors chip is capable of quantitative analysis exosomes markers. Additionally, the change of current signals due to CD9 and CD63 proteins are higher than that of CD81 protein, indicating that the current response caused by immunoreaction is related to the type of exosomes marker. 

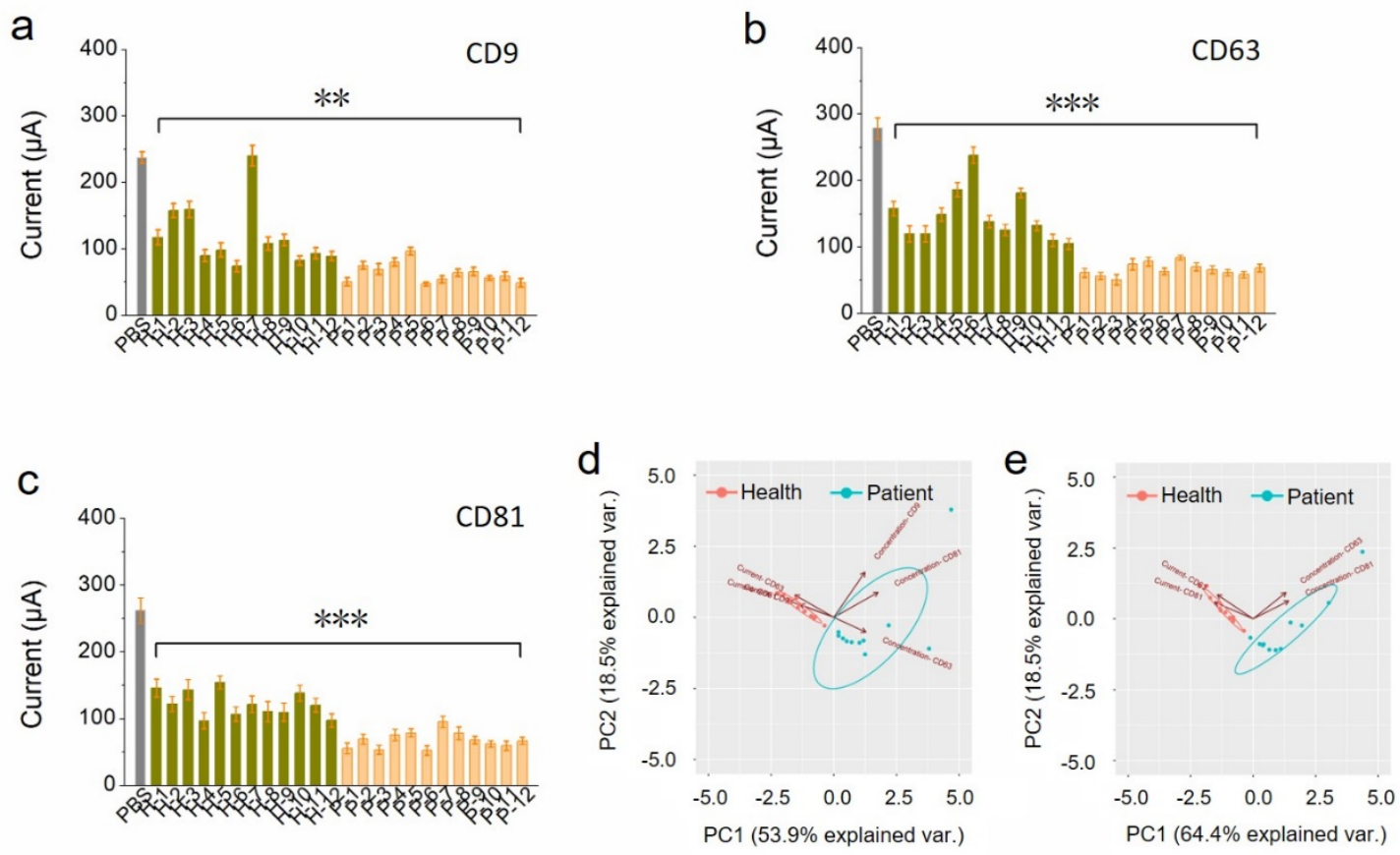

Figure 5. Profiling human lung cancer blood samples through ELB. a, b, c, Analysis of exosomes secreted by lung carcinoma cells in clinical human blood samples. Error bars represent mean+/-SD of 3-5 independent experiments. Differences with ${ }^{* *} \mathrm{P}<0.005$ and ${ }^{* * *} \mathrm{P}<0.0001$ were considered statistically significant by $\mathrm{T}$-test. $\mathbf{d}, \mathbf{e}$, Scores plots of PC1 vs. PC2 produced using data from clinical human blood assay.

\section{Direct exosome quantification from cultures of human non-small cell lung cancer}

We challenged our platform for the detection the exosomes from culture supernatant of human non-small cell lung cancer cell line A54. Bicinchoninic Acid (BCA) Protein Assay Kit was used for determining the concentration of exosomes extracted from cell supernatant (see Methods), and the measured concentration was $100 \mu \mathrm{g} / \mathrm{mL}$. For quantification, different concentrations $\left(5 \times 10^{4} \sim 1 \times 10^{-5}\right.$ $\mathrm{ng} / \mathrm{mL}$ ) of exosomes were obtained by dilution and added into the microsensors chip. The dose-dependent current signals were measured (Fig. 4g-4i), showing that the output signal was correlated with the concentration of exosomes. The developed microsensors chip can effectively detect exosomes in the concentration range from $\mathrm{ng} / \mathrm{mL}$ to $\mathrm{pg} / \mathrm{mL}$. The limit of detection (LOD) was evaluated to be $10 \mathrm{fg} / \mathrm{mL}$ for CD9, $1 \mathrm{fg} / \mathrm{mL}$ for CD63 and $1 \mathrm{fg} / \mathrm{mL}$ for CD81, respectively. The LOD was superior to that of previous reports ${ }^{30-33}$. These results confirm the capability of the microchip to probe exosomes in complex samples.

\section{Analyzing human lung cancer blood samples}

To demonstrate the practical application of our developed microsensor chip, human blood samples were analyzed. Twenty-four clinical blood samples were collected, twelve of them were from healthy individuals $(\mathrm{H} 1-\mathrm{H} 12)$ and the other twelve were samples of cancer patients (P1-P12). All the samples were diluted to 15-fold in PBS solution and the current signal generated by blank PBS solution was also quantified as an experimental control. We found that the current response of clinical blood samples from healthy individuals was higher than the current from cancer patients (Fig. 5a, b), which demonstrates that exosomes from cancer patients express higher levels of CD9, CD63 and CD81 than healthy subjects. Specifically, the electrodes immobilized with Anti-CD63 and Anti-CD81 could 
effectively distinguish the cancer patients and healthy controls, while three of cancer samples and one in healthy group displayed the abnormal expression in the testing results of CD9. Since surface proteins on exosomes carry information about their tissues of origin, they may have different expression profiles on exosomes, thus inducing some variation in quantification of exosomes ${ }^{15}$.

To enable accurate data processing, we utilized principal component analysis (PCA), which is a statistical analysis method utilized to investigate major patterns in data sets ${ }^{34}$, to analyze variances in each collected sample and grouping them by similarity. The concentration of exosomes was quantified according to the fitted curves in Figure 4. Figure $5 \mathrm{~d}$ and $5 \mathrm{e}$ present the PCA scores plots of human serum samples. When we selected the protein combination of all three markers, the variance levels in PC1 and PC2 were $53.9 \%$ and $18.7 \%$, respectively. However, as the protein combination was defined as CD63 and CD81, the clusters of exosome data could be well-distinguished to cancerous group and health control, demonstrating a robust performance for cancer detection.

\section{Discussion}

Using such rational and bottom-up design approach, we demonstrated a generalizable strategy to encode desirable sensing functions into nano-structured materials. Under the synergistic effect of different components within the hybrid nanostructure, a multiplexed, electrochemical liquid biopsy (ELB) platform was developed and tested with human blood samples, providing accurate clinical insights into cancer severity. Furthermore, the design of this platform technology is modular and logical, therefore serving as a general strategy for the detection of other biomarkers.

Owing to the cost-effectiveness and simplicity of electrochemical sensing comparing with the optical orthologs, electrochemical biosensor represents a promising class of point-of-care systems as exemplified by the gold-standard, commercialized glucose sensor ${ }^{14,35-44}$. Despite significant advances in sensing chemistry ${ }^{44-52}$, challenges remained in electrochemical immunosensors have significantly hindered our capability to assemble an integrated sensing system capable of direct, high-sensitivity and high-accuracy profiling of biomarkers through immunochemistry. First, most of the proteins and nucleic acids are not redox active $\mathrm{A}^{42,53}$. Therefore, to achieve quantification on an electrochemical surface, characterizing change of surface impedance or constructing redox active molecular interface is required with inevitable introduction of external redox molecules ${ }^{14,40}$. By introducing solid-phase redox active species, we encoded the capability of signal transduction directly onto the electrode materials, providing a generalizable redox-active material interface. Second, surface modification has been significantly investigated and optimized in order to achieve immobilization of recognition elements with optimal conformations ${ }^{54,55}$. By forming homogeneous $3 \mathrm{D}$ nanopillars and introducing ZIF-90 with active $\mathrm{CHO}$ groups onto the electrode surface, we were able to immobilize conformational ideal and biological active antibody directly onto the electrode without tailoring the surface chemistry, delivering a simple fabrication method for immunosensor. The functionalized nanopillar is also capable of target sorting from complex matrix. Furthermore, the 3D nanostructure built with high-electron conduction materials also provide sufficient electron transfer and anti-fouling properties ${ }^{11}$. Finally, to enable a high-accuracy sensing system or a multi-use sensor, capability of multiplexed analysis is desirable ${ }^{50,56}$. Therefore, multiplexed microfluidic channels were designed to accommodate sensor fabrication and sample input. By examining the overall sensor performance with clinical samples, we 
confirmed the capability of the rationally engineered ELB platform on profiling and differentiating of physiologically relevant markers. These results indicate that our rational engineering design through encoding sensing function into material interface is a general approach for the developments of electrochemical biosensors.

\section{Methods}

\section{Synthesis of $2 \mathrm{H}-\mathrm{MoS}_{2}$}

Ultrathin $\mathrm{MoS}_{2}$ with $2 \mathrm{H}$-phase was synthesized by sonication exfoliation of bulk $\mathrm{MoS}_{2}$ in liquid nitrogen ${ }^{6}$. Typically, $0.1 \mathrm{~g} \mathrm{MoS}_{2}$ was dispersed into $20 \mathrm{~mL} \mathrm{KOH}(1 \mathrm{~g})$ solution. The mixture was then stirred vigorously for $24 \mathrm{~h}$ at $80^{\circ} \mathrm{C}$. After being cooled down to room temperature, liquid nitrogen was quickly poured into the mixture to quench the reaction. The quench process was performed at least four times to effectively prevent the van der Waals forces binding in bulk $\mathrm{MoS}_{2}$. The mixture was then sonicated for $3 \mathrm{~h}$ and collected by vacuum filtration. The ultrathin $2 \mathrm{H}-\mathrm{MoS}_{2}$ was finally obtained after drying in vacuum at $60^{\circ} \mathrm{C}$ overnight.

\section{Synthesis of $\mathrm{MoS}_{2} / \mathrm{ZnO} / \mathrm{ZIF}-90$}

The synthesis of $\mathrm{ZnO@MoS} 2$ was carried out through seed-assisted growth strategy. First, the obtained $2 \mathrm{H}-\mathrm{MoS}_{2}$ was dispersed into ethanol $(0.4 \mathrm{mg} / \mathrm{mL})$, and then $1.6 \mathrm{~mL}$ of obtained suspension was deposited on a clean silicon wafer with a size of $1 \times 1 \mathrm{~cm}^{2} .250 \mu \mathrm{L}$ of $\mathrm{Zn}(\mathrm{Ac}$ )/ /ethanol solution (5 $\mathrm{mM}$ ) was then dropped onto $2 \mathrm{H}-\mathrm{MoS}_{2}$. After drying at room temperature, the silicon wafer was transferred to a tube furnace and calcinated for $20 \mathrm{~min}$ at $350{ }^{\circ} \mathrm{C}$. The above silicon wafer loaded with $2 \mathrm{H}-\mathrm{MoS}_{2}$ and $\mathrm{ZnO}$ seeds was laid face down on a solution containing $\left.25 \mathrm{mM} \mathrm{Zn(NO}\right)_{3} \cdot 6 \mathrm{H}_{2} \mathrm{O}, 25 \mathrm{mM}$ hexamethylene tetramine, $6 \mathrm{mM}$ polyethylene imine and $80 \mathrm{~mL}$ deionized water. The solution was sealed in a $100 \mathrm{~mL}$ premium bottle (Schott Duran) and kept at $90^{\circ} \mathrm{C}$ for $5 \mathrm{~h}$. After the reaction was completed, the silicon wafer was washed with ethanol and deionized water for three times. ZIF-90 films were grown in situ on $\mathrm{ZnO@MoS} 2$ by using $\mathrm{ZnO}$ nanopillars as self-sacrificing template and precursor to directly react with the vapor phase of imidazole-2-formaldehyde. The silicon wafer loaded with $\mathrm{MoS}_{2} / \mathrm{ZnO}$ was suspended in a $100 \mathrm{~mL}$ premium bottle with $0.5 \mathrm{~g}$ imidazole-2-formaldehyde at the bottom. The vial was kept at $150{ }^{\circ} \mathrm{C}$ for $30 \mathrm{~min}$. After the completion of the reaction, the silicon wafer was quickly transferred to a $150{ }^{\circ} \mathrm{C}$ vacuum oven and kept for $10 \mathrm{~min}$ to remove impurities. The $\mathrm{MoS}_{2} / \mathrm{ZnO} / \mathrm{ZIF}-90$ was finally obtained after cooling down to room temperature.

\section{Isolation and Concentration Determination of Exosome}

Exosomes were harvested from supernatant of human non-small cell lung cancer cell A549 using a QIAGEN Assay kit, Article No. P76064. Briefly, the supernatant was filtered before freezing and then mixed with an equal volume of Buffer XBP evenly and warmed up to room temperature. The supernatant/XBP mixture was added to an exoEasy spin column and centrifuged at $500 \mathrm{~g}$ for $1 \mathrm{~min}$. The flow-through fluid was discarded and the column was placed back into the same collection tube. $10 \mathrm{~mL}$ buffer XWP was added and centrifuged at $5000 \mathrm{~g}$ for $5 \mathrm{~min}$ to remove residual buffer. The spin column was then transferred to a fresh collection tube. $400 \mu \mathrm{L}$ buffer XE was added to the membrane and incubated for $1 \mathrm{~min}$ followed by centrifugation at $500 \mathrm{~g}$ for $5 \mathrm{~min}$ to collect the eluate, the eluate was then re-applied to the exoEasy spin membrane and incubated for $1 \mathrm{~min}$. Centrifugation at $5000 \mathrm{~g}$ for 5 min was performed to collect the eluate and the finally extracted exosomes were transferred to an appropriate tube for concentration determination. 
The concentration of extracted exosomes was determined using a Beyotime BCA protein Assay Kit, Article No. P0012. An appropriate amount of BCA working solution was prepared according to the instruction and placed at room temperature for $24 \mathrm{~h}$ to keep it stable. PBS buffer was used to completely dissolve the protein standard and maintain its concentration at $0.5 \mathrm{mg} / \mathrm{mL}$. A series of increasing volume of standard/PBS solution ranging from $0 \mu \mathrm{L}$ to $20 \mu \mathrm{L}$ was added to the standard plate of 96 wells respectively. Additionally, $20 \mu \mathrm{L}$ of exosomes suspension was added to the sample plate of 96 wells. $200 \mu \mathrm{L}$ of BCA working solution was added to each well and placed at $37{ }^{\circ} \mathrm{C}$ for 30 $\mathrm{min}$. The microplate reader was used to measure the light intensity under $562 \mathrm{~nm}$ wavelength light and the concentration of exosomes was calculated according to the standard curve.

\section{Fabrication of Electrochemical sensor chip}

The microfluidic electrochemical sensor chip was configured with a modified five-electrode system and sealed with polydimethylsiloxane (PDMS) layer. The electrochemical electrodes including three working electrodes (WE) as well as their shared reference electrode and counter electrode were screen-printed on polyethylene terephthalate (PET) substrate. Both working electrodes and counter electrode were printed with carbon and silver conductive ink, the reference electrode was printed with silver/silver chloride $\left(\mathrm{Ag} / \mathrm{AgCl}\right.$ ) ink. The as-prepared $\mathrm{MoS}_{2} / \mathrm{ZnO} / \mathrm{ZIF}-90$ material $(4 \mathrm{mg}$ ) was dispersed into the mixture of ethanol $(100 \mu \mathrm{L})$ and Nafion 117 solution $(25 \mu \mathrm{L})$ forming a homogeneous ink, then locally deposited onto the surface of working electrodes. A 3D-printed template assisted method was used to fabricate PDMS microchannels, and the resin template was printed via a high-resolution DLP/SLA 3D printer (nanoArch S140). The PDMS layer was peeled off from the resin template and then bonded to PET sensor chip by air plasma treatment using a PDC-002-HP Plasma Cleaner (HARRICK PLASMA) under $45 \mathrm{~W}$ of treating power in vacuum for $90 \mathrm{~s}$.

\section{Electrochemical Test}

Electrochemical tests were operated on an electrochemical workstation (VSP-300, BioLogic, France). Electrochemical impedance spectroscopy (EIS) was performed in a solution containing $6.7 \mathrm{mM} \mathrm{MgCl}$, $7.6 \mathrm{mM}$ potassium ferrocyanide, $6.7 \mathrm{mM}$ potassium ferricyanide and $300 \mathrm{~mL}$ deinoized water with frequency ranging from $10^{5}$ to $10^{-2} \mathrm{~Hz}$ by applying amplitude of $10 \mathrm{mV}$. The DPV test was performed on the microsensor chip in a potential interval of $0-0.4 \mathrm{~V} \mathrm{vs.} \mathrm{Ag} / \mathrm{AgCl}$ and $\mathrm{CV}$ test was performed in a potenial interval of $-0.6 \mathrm{~V}-0.0 \mathrm{~V}$ and $0.0 \mathrm{~V}-0.6 \mathrm{~V}$ to evaluate the electrochemical response signal.

\section{Electrochemical immunoassays}

Since exosome marker is not exclusive, some of the popular antibodies targeted against exosome associated antigens are cluster of differentiation. Here, three typical exosomes markers (CD9, CD63 and CD81) are employed for the quantification of exosomes in biological samples. In addition, the developed microsensor chip allows multiplex analysis of exosomes from a single sample, which would offer high accuracy and great efficiency through the mutual validation of the acquired data for multiple markers. Firstly, Anti-CD9 antibody/PBS (10 $\mu \mathrm{g} / \mathrm{mL})$, Anti-CD63 antibody/PBS (10 $\mu \mathrm{g} / \mathrm{mL})$, Anti-CD81 antibody/PBS $(10 \mu \mathrm{g} / \mathrm{mL})$ solution were separately injected into different channels through inlet 1,2 and 3 , and then incubated at $4{ }^{\circ} \mathrm{C}$ for $12 \mathrm{~h}$. After that, the microchannels were washed three times with wash buffer (PBS+0.05\% Tween 20) to remove the unbonded antibodies. In order to perform the assays, the WEs were then blocked for $1 \mathrm{~h}$ at room temperature with $150 \mu \mathrm{L}$ of $5 \%$ BSA in PBS, and 
again washed three times with wash buffer. The assays were firstly verified by using recombinant $C D$ $(9,63$ and 81$)$ proteins, and then calibrated using culture supernatant from human non-small cell lung cancer cell line A54. All the measurements were repeated at least three times to ensure reproducibility. Clinical blood samples were collected at Tongji Hospital and analyzed in duplicates.

\section{References}

1 Dong, E., Du, H. \& Gardner, L. An interactive web-based dashboard to track COVID-19 in real time. Lancet Infectious Diseases 20, 533-534 (2020).

2 Miller, I. F., Becker, A. D., Grenfell, B. T. \& Metcalf, C. J. E. Disease and healthcare burden of COVID-19 in the United States. Nature Medicine 26, 1212-1217 (2020).

3 Al-Quteimat, O. M. \& Amer, A. M. The impact of the COVID-19 pandemic on cancer patients. American journal of clinical oncology (2020).

4 Russano, M. et al. Liquid biopsy and tumor heterogeneity in metastatic solid tumors: the potentiality of blood samples. Journal of Experimental \& Clinical Cancer Research 39 (2020).

5 Palmirotta, R. et al. Liquid biopsy of cancer: a multimodal diagnostic tool in clinical oncology. Therapeutic Advances in Medical Oncology 10, 1-24 (2018).

6 Ying, Z. et al. Phase-Regulated Sensing Mechanism of MoS2 Based Nanohybrids toward Point-of-Care Prostate Cancer Diagnosis. Small 16 (2020).

7 Liu, J. et al. Two-dimensional metal-organic frameworks nanosheets: Synthesis strategies and applications. Inorganica Chimica Acta 483, 550-564 (2018).

8 Migneault, I., Dartiguenave, C., Bertrand, M. J. \& Waldron, K. C. Glutaraldehyde: behavior in aqueous solution, reaction with proteins, and application to enzyme crosslinking. Biotechniques 37, 790-+ (2004).

9 Hui, J. Z. et al. Facile Method for the Site-Specific, Covalent Attachment of Full-Length IgG onto Nanoparticles. Small 10, 3354-3363 (2014).

10 lijima, M. \& Kuroda, S. i. Scaffolds for oriented and close-packed immobilization of immunoglobulins. Biosensors \& Bioelectronics 89, 810-821 (2017).

11 Sabaté del Río, J., Henry, O. Y. F., Jolly, P. \& Ingber, D. E. An antifouling coating that enables affinity-based electrochemical biosensing in complex biological fluids. Nature Nanotechnology 14, 1143-1149, doi:10.1038/s41565-019-0566-z (2019).

12 Welch, E. C., Powell, J. M., Clevinger, T. B., Fairman, A. E. \& Shukla, A. Advances in Biosensors and Diagnostic Technologies Using Nanostructures and Nanomaterials. Advanced Functional Materials 31 (2021).

13 Beit-Yannai, E., Tabak, S. \& Stamer, W. D. Physical exosome:exosome interactions. Journal of Cellular and Molecular Medicine 22, 2001-2006, doi:https://doi.org/10.1111/jcmm.13479 (2018).

14 Furst, A. L. \& Francis, M. B. Impedance-Based Detection of Bacteria. Chemical Reviews 119, 700-726, doi:10.1021/acs.chemrev.8b00381 (2019).

15 Wu, D. et al. Profiling surface proteins on individual exosomes using a proximity barcoding assay. Nature Communications 10 (2019).

16 Xie, F. et al. Extracellular Vesicles in Cancer Immune Microenvironment and Cancer Immunotherapy. Advanced Science 6, doi:10.1002/advs.201901779 (2019).

17 Mohammadniaei, M. et al. Electrochemical Biosensor Composed of Silver Ion-Mediated 
dsDNA on Au-Encapsulated Bi2Se3 Nanoparticles for the Detection of H2O2 Released from Breast Cancer Cells. Small 14 (2018).

18 Zhou, Y. et al. In Situ Electrodeposited Synthesis of Electrochemiluminescent Ag Nanoclusters as Signal Probe for Ultrasensitive Detection of Cyclin-D1 from Cancer Cells. Analytical Chemistry 89, 6787-6793 (2017).

19 Chen, W. et al. Schiff-base reaction induced selective sensing of trace dopamine based on a Pt41Rh59 alloy/ZIF-90 nanocomposite. Nanotechnology 30 (2019).

20 Fan, H. et al. MOF-in-COF molecular sieving membrane for selective hydrogen separation. Nature Communications 12 (2021).

21 Morris, W., Doonan, C. J., Furukawa, H., Banerjee, R. \& Yaghi, O. M. Crystals as molecules: Postsynthesis covalent functionalization of zeolitic imidazolate frameworks. Journal of the American Chemical Society 130, 12626-+ (2008).

22 Zhang, F.-M. et al. Postsynthetic Modification of ZIF-90 for Potential Targeted Codelivery of Two Anticancer Drugs. Acs Applied Materials \& Interfaces 9, 27332-27337 (2017).

23 Jiao, L. et al. Single-atom catalysts boost signal amplification for biosensing. Chemical Society Reviews 50, 750-765 (2021).

24 Nam, J. et al. Supramolecular Peptide Hydrogel-Based Soft Neural Interface Augments Brain Signals through a Three-Dimensional Electrical Network. Acs Nano 14, 664-675 (2020).

25 Soleymani, L., Fang, Z., Sargent, E. H. \& Kelley, S. O. Programming the detection limits of biosensors through controlled nanostructuring. Nature Nanotechnology 4, 844-848, doi:10.1038/nnano.2009.276 (2009).

26 Chowdhury, A. D., Takemura, K., Li, T.-C., Suzuki, T. \& Park, E. Y. Electrical pulse-induced electrochemical biosensor for hepatitis E virus detection. Nature Communications 10 (2019).

27 Pang, X. et al. Visible Light-Driven Self-Powered Device Based on a Straddling Nano-Heterojunction and Bio-Application for the Quantitation of Exosomal RNA. Acs Nano 13, 1817-1827 (2019).

28 Mehennaoui, S., Poorahong, S., Jimenez, G. C. \& Siaj, M. Selection of high affinity aptamer-ligand for dexamethasone and its electrochemical biosensor. Scientific Reports 9 (2019).

29 Yan, M. et al. Ultrasensitive Immunosensor for Cardiac Troponin I Detection Based on the Electrochemiluminescence of 2D Ru-MOF Nanosheets. Analytical Chemistry 91, 10156-10163 (2019).

30 Jiang, Y. et al. Aptamer/AuNP Biosensor for Colorimetric Profiling of Exosomal Proteins. Angewandte Chemie (International ed. in English) 56, 11916-11920 (2017).

31 Suwatthanarak, T. et al. Microfluidic-based capture and release of cancer-derived exosomes via peptide-nanowire hybrid interface. Lab on a chip 21, 597-607 (2021).

32 Yoshioka, Y. et al. Ultra-sensitive liquid biopsy of circulating extracellular vesicles using ExoScreen. Nat Commun 5, 3591 (2014).

33 Zhang, R. et al. Label-Free Electrochemical Sensor for CD44 by Ligand-Protein Interaction. Anal Chem 91, 7078-7085 (2019).

34 Wold, S., Esbensen, K. \& Geladi, P. Principal component analysis. Chemometrics and intelligent laboratory systems 2, 37-52 (1987).

35 Kelley, S. O. et al. Advancing the speed, sensitivity and accuracy of biomolecular detection using multi-length-scale engineering. Nature Nanotechnology 9, 969-980, 
doi:10.1038/nnano.2014.261 (2014).

6 Wang, J. Electrochemical Glucose Biosensors. Chemical Reviews 108, 814-825, doi:10.1021/cr068123a (2008).

37 Song, Y., Mukasa, D., Zhang, H. \& Gao, W. Self-Powered Wearable Biosensors. Accounts of Materials Research 2, 184-197, doi:10.1021/accountsmr.1c00002 (2021).

$38 \mathrm{Li}, \mathrm{D}$., Song, S. \& Fan, C. Target-responsive structural switching for nucleic acid-based sensors. Accounts of Chemical Research 43, 631-641 (2010).

39 Lubin, A. A. \& Plaxco, K. W. Folding-based electrochemical biosensors: the case for responsive nucleic acid architectures. Accounts of chemical research 43, 496-505 (2010).

Zamani, M., Furst, A. L. \& Klapperich, C. M. Strategies for Engineering Affordable Technologies for Point-of-Care Diagnostics of Infectious Diseases. Accounts of Chemical Research 54, 3772-3779 (2021).

41 Wu, Y., Tilley, R. D. \& Gooding, J. J. Challenges and solutions in developing ultrasensitive biosensors. Journal of the American Chemical Society 141, 1162-1170 (2018).

42 Dai, Y. \& Liu, C. C. Recent Advances on Electrochemical Biosensing Strategies toward Universal Point-of-Care Systems. Angewandte Chemie International Edition 58, 12355-12368, doi:https://doi.org/10.1002/anie.201901879 (2019).

43 Dai, Y. et al. An Integrated Multi-Function Heterogeneous Biochemical Circuit for HighResolution Electrochemistry-Based Genetic Analysis. Angewandte Chemie International Edition 59, 20545-20551 (2020).

44 Dai, Y., Wu, Y., Liu, G. \& Gooding, J. J. CRISPR Mediated Biosensing Toward Understanding Cellular Biology and Point-of-Care Diagnosis. Angewandte Chemie 132, 20938-20950 (2020). Kaminski, M. M., Abudayyeh, O. O., Gootenberg, J. S., Zhang, F. \& Collins, J. J. CRISPR-based diagnostics. Nature Biomedical Engineering 5, 643-656, doi:10.1038/s41551-021-00760-7 (2021).

46 Sempionatto, J. R., Jeerapan, I., Krishnan, S. \& Wang, J. Wearable Chemical Sensors: Emerging Systems for On-Body Analytical Chemistry. Analytical Chemistry 92, 378-396, doi:10.1021/acs.analchem.9b04668 (2020).

47 Mahshid, S. S., Camiré, S., Ricci, F. \& Vallée-Bélisle, A. A Highly Selective Electrochemical DNA-Based Sensor That Employs Steric Hindrance Effects to Detect Proteins Directly in Whole Blood. Journal of the American Chemical Society 137, 15596-15599, doi:10.1021/jacs.5b04942 (2015).

48 Baranda Pellejero, L. et al. Using antibodies to control DNA-templated chemical reactions. Nature Communications 11, 6242, doi:10.1038/s41467-020-20024-3 (2020).

49 Yang, F., Li, Q., Wang, L., Zhang, G.-J. \& Fan, C. Framework-nucleic-acid-enabled biosensor development. ACS sensors 3, 903-919 (2018).

50 Dincer, C., Bruch, R., Kling, A., Dittrich, P. S. \& Urban, G. A. Multiplexed Point-of-Care Testing - XPOCT. Trends in Biotechnology 35, 728-742, doi:https://doi.org/10.1016/i.tibtech.2017.03.013 (2017).

51 Dai, Y. et al. Exploring the Trans-Cleavage Activity of CRISPR-Cas12a (cpf1) for the Development of a Universal Electrochemical Biosensor. Angewandte Chemie 131, 17560-17566 (2019).

52 Kishi, J. Y., Schaus, T. E., Gopalkrishnan, N., Xuan, F. \& Yin, P. Programmable autonomous synthesis of single-stranded DNA. Nature chemistry 10, 155-164 (2018). 
53 Das, J. et al. Reagentless biomolecular analysis using a molecular pendulum. Nature Chemistry 13, 428-434, doi:10.1038/s41557-021-00644-y (2021).

54 Campuzano, S., Yáñez-Sedeño, P. \& Pingarrón, J. M. Tailoring sensitivity in electrochemical nucleic acid hybridization biosensing: Role of surface chemistry and labeling strategies. ChemElectroChem 6, 60-72 (2019).

55 Furst, A., Landefeld, S., Hill, M. G. \& Barton, J. K. Electrochemical Patterning and Detection of DNA Arrays on a Two-Electrode Platform. Journal of the American Chemical Society 135 , 19099-19102, doi:10.1021/ja410902j (2013).

56 Heggestad, J. T. et al. Multiplexed, quantitative serological profiling of COVID-19 from blood by a point-of-care test. Science Advances 7, eabg4901, doi:doi:10.1126/sciadv.abg4901 (2021).

\section{Acknowledgements}

This work was funded by National Key Research and Development Program of China (No. 2021Yfb3200804), Shanghai "Road and Belt" International Cooperation Project (No. 19520744200), Key Basic Research Program of Science and Technology Commission of Shanghai Municipality (20JC1415300). Y.Z. appreciates the financial support of Henan International Joint Research Laboratory of Nanocomposite Sensing Materials. C.L and Y.D appreciate the financial support of Wallace R. Persons Foundation.

\section{Funding}

National Key Research and Development Program of China (No. 2021Yfb3200804)

Key Basic Research Program of Science and Technology Commission of Shanghai Municipality (20JC1415300)

\section{Author contributions}

Y.Z. and Y.D. conceptualized the project, interpreted the results, and wrote the manuscript. H.Z. and Z.Y. performed the experiments and analyzed the data. X.G., W.C. and Y.Z. participated in the experiments on isolation and concentration determination of exosomes. All authors revised the manuscript.

\section{Competing interests}

The authors declare no competing interests.

\section{Data and materials availability}

All data and materials are available from corresponding author upon reasonable request.

\section{Supplementary Materials}

Materials and Methods, Supplementary Figures 